\title{
Liquid Drop Model for Investigating the Outer Crust of Neutron Star
}

\author{
Feni Fitrishia $^{1}$, Eko Tri Sulistyani ${ }^{2 *}$, Romy Hanang Setya Budhi ${ }^{2}$ \\ ${ }^{1}$ Physics Department, ${ }^{2}$ Research Group of Astrophysics, Cosmology and Mathematical Physics, Faculty of Mathematics and Natural Science \\ Gadjah Mada University, Bulaksumur, Caturtunggal, Yogyakarta 55281, Indonesia. Tel. +62-274-6492599, Fax. +62-274-565223 \\ Email: feni.fitrishia@mail.ugm.ac.id ${ }^{1}$, sulistyani@ugm.ac.id ${ }^{2 *}$, romyhanang@ugm.ac.id ${ }^{2}$
}

\begin{abstract}
The properties of outer crust of cooling neutron star by using Liquid Drop Model approximation has been studied. This mass model is used to derive the properties of outer crust of neutron star matter such as total energy of system, equation of state (relationship between pressure and density), and composition of the outer crust. The properties of the outer crust are studied under the assumption that neutron star has created for long time and the matter in neutron star is in its ground state. The composition of the outer crust consists of ions $Z$ arranged in a lattice and free electrons $e$, so that the total energy of the system consists of three contributions: the mass-energy of the nuclear, the energy of electrons and lattice. The equation of state (the relationship between pressure and density) shows that the pressure increases with increasing density in the outer crust layer. With a simple model based on liquid drop model, it is known that the outer crust is composed of nuclei which are at $\mathrm{Z} \approx 20-50$, with ${ }^{56} \mathrm{Fe}$ core at the upper layer, and ${ }^{154} \mathrm{Cd}$ core at the lowermost layer of the outer crust.
\end{abstract}

Keywords: Liquid drop model, Neutron star, Outer crust

\section{INTRODUCTION}

The neutron star is one of dense objects which has central density between 5 and 10 times normal nuclear density $\rho_{0}$, where $\rho_{0} \cong 2,8 \times 10^{14} \mathrm{~g} \mathrm{~cm}^{-3}$ (Lattimer and Prakash, 2004). Neutron stars are remnants of massive stars that explode at its final stage of their evolution. This explosion is known as supernova. Supernova is caused by a massive star which all the nuclear fuel has been exhausted infusion reactions, so that pressure of its core cannot compensate the gravitational pressure, or can be called gravitational collapse (Haensel et al., 2007). A neutron star can be subdivided into two main regions: the core and the envelope. The core can be subdivided into the outer core and inner core. The envelope can be subdivided into the atmosphere, the outer crust, and the inner crust. By using semiempirical mass formula, nuclear energy in the outer crust can be calculated.

The outer crust is a layer of neutron star extends from the layer of the density $\rho \cong 10^{4} \mathrm{~g} \mathrm{~cm}^{-3}$ (ionization) to the $\rho \cong 10^{11} \mathrm{~g} \mathrm{~cm}^{-3}$ (neutron drip). Its matter consists of ions $Z$ which arranged in crystal Coulomb and free electrons $e$. Outer crust is one of layer of neutron stars which important to study. Interpretation of many observations for neutron stars phenomena like pulsar perturbations, toroidal oscillation in soft gamma repeater and in initial cooling process will related strongly with composition of outer crust (Chamel, 2007).

Quantity of nuclear energy is not known experimentally for the very neutron-rich nuclei produced above $10^{11} \mathrm{~g} \mathrm{~cm}^{-3}$ and must be inferred theoretically. This is usually done by means of a semiempirical mass formula, at least up to the point of neutron drip (Shapiro and Teukolsky, 1983). Semi- empirical mass formula is used to calculate nuclear mass in the outer crust of non-accreting neutron star. Semi-empirical mass formula is developed by Weizsäcker (1935) on the basis of the liquid-drop analogy for nuclear matter, suggested by Bohr (Meyerhof, 1967). The nucleus is a system constituted by a strongly interacting of protons and neutrons, also considered as the same particle, namely nucleon (RocaMaza, 2010). The outer crust will be reviewed by using BPS model which have derived by Baym, Pethick and Sutherland (1971) and using toy model which have developed by Roca-Maza and Piekarewicz (2008). This work will be due to study properties of the outer crust such as total energy of system, equation of state (relationship between pressure and density), and how to apply liquid drop model for investigating composition of the matter in the outer crust.

\section{MATERIALS AND METHODS}

In the present section, we review the most important features of the formalism necessary to describe the outer crust of a non accreting neutron star. We closely follow the BPS model introduced by Baym, Pethick, and Sutherland (Baym, 1971) and using toy model which have developed by Roca-Maza and Piekarewicz (2008). To a first approximation, the matter in neutron stars is in good approximation as a cold system $(T \approx 0)$; and it may be assumed to be in nuclear equilibriumthat is, its energy cannot be lowered by changing its constituents via strong, weak, or electromagnetic interactions. Outer crust consists of ions $Z$ which arranged in crystal Coulomb and free electrons $e$. The principal contributions to the energy of matter in this regime are the energy density of nuclear, the energy 
density of free electron, and the energy density of lattice. Liquid drop model will be used to calculate nuclear energy and free electron is assumed relativistic (Roca-Maza et al., 2011).

\section{RESULTS AND DISCUSSION}

The outer crust is composed by nuclei arranged in a Coulomb lattice and embedded in a uniform free Fermi gas of electrons. According to BPS model, to find out the ground state of matter at the density before neutron drip can be done by calculating the total energy density of the outer crust layer. The total energy of the system before neutron drip is (Roca-Maza et al., 2011):

$\varepsilon_{\text {tot }}\left(A_{s} Z ; n_{b}\right)=\varepsilon_{n}+\varepsilon_{\theta}+\varepsilon_{L}$.

Term $\varepsilon_{n}, \varepsilon_{\varepsilon}, \varepsilon_{L}$ at the right of equation 3.1 respectively is the energy density of nuclear, energy density of free electron and energy density of lattice.

\section{Energy Density of Nuclear}

Nuclear contribution $\varepsilon_{n n}$ to the total energy per nucleon is (Roca-Maza et al., 2011):

$\varepsilon_{n}=E(A, Z) / A$ with

$E(A, Z)=\left(m_{p} c^{2} Z+m_{n} c^{2}(A-Z)\right)-B(A, Z)$,

where $B(A, Z)$ is binding energy of nucleons in nucleus:

$B(A, Z)=a_{v} A-a_{s} A^{\frac{2}{3}}-a_{c} \frac{Z(Z-1)}{A^{\frac{1}{3}}}-a_{a} \frac{(A-2 Z)^{2}}{A}$

so equation 3.2 can be written as:

$\varepsilon_{n}(A, Z)=\frac{E}{A}=m_{p} \frac{Z}{A}+m_{n}-m_{n} \frac{Z}{A}-a_{v}+\frac{a_{s}}{A^{\frac{1}{3}}}+a_{c} \frac{Z^{2}}{A^{\frac{4}{3}}}+a_{a} \frac{(A-2 Z)^{2}}{A^{2}}$

\section{Energy Density of Electron}

The electronic contribution at the densities of interest is modeled as a degenerate free Fermi gas (Roca-Maza et al., 2011).

$\varepsilon_{\theta}\left(A, Z ; n_{b}\right)=\frac{E_{\theta}}{n_{b}}=\frac{1}{n_{b} \pi^{2}} \int_{0}^{p_{F g}} p^{2} \sqrt{p^{2} c^{2}+m_{g}^{2} c^{4}} d p$,

$\varepsilon_{\varepsilon}=\frac{p_{F \varepsilon}}{8 \pi^{2} n_{b}}\left(2 p_{F \varepsilon}^{2}+m_{\varepsilon}^{2}\right) \sqrt{p_{F \theta}^{2}+m_{\varepsilon}^{2}}-\frac{m_{\varepsilon}^{4}}{8 \pi^{2} n_{b}} \ln \left(\frac{p_{F \varepsilon}+\sqrt{p_{F \theta}^{2}+m_{\varepsilon}^{2}}}{m_{\varepsilon}}\right)$,

By using dimensionless parameter $x_{F}$ and $y_{F}$,

$x_{F}=\frac{p_{F e}}{m_{e} c}$ and $y_{F}=\frac{E_{F \theta}}{m_{e}}=\sqrt{1+x_{F}^{2}}$.

equation (3.5) can be written as:

$\varepsilon_{\theta}=\frac{m_{\theta}^{4}}{8 \pi^{2} m_{b}}\left(x_{F} y_{F}\left(x_{F}^{2}+y_{F}^{2}\right)-\ln \left(x_{F}+y_{F}\right)\right)$.

where $\varepsilon_{g}, m_{g}, p_{F \theta}$ respectively are energy density of free electron, mass of electron, Fermi momentum of electron, and $n_{b}$ is baryon density $\left(n_{b}=A_{\text {tot }} / V\right)$.

\section{Energy Density of Lattice}

At very low density, matter in its ground state consists of ${ }^{56} \mathrm{Fe}$ nuclei, arranged in a lattice so as to minimize their Coulomb interaction energy (Baym et al., 1971).
In the 'Wigner-Seitz approximation' the gas electron is assumed to be divided into neutral spheres of radius $r$, about each nucleus, which contains the $Z$ electrons closest to the nucleus. The total energy of any one sphere is the sum of the Coulomb energies due to electron-electron $(e-e)$ interactions and electron-ion $(e$ - i) interactions (Shapiro and Teukolsky, 1983). Energy density of lattice is given by (Roca-Maza and Piekarewicz 2008; Sharma et al., 2015):

$\varepsilon_{L}\left(A_{s} Z ; n_{b}\right)=-\left(3,40665 \times 10^{-\mathrm{a}}\right) \frac{Z^{2}}{A^{4 / 3}} p_{F}$

$\varepsilon_{L}\left(A_{v} Z_{s} n_{b}\right)=-C_{L} \frac{Z^{2}}{A^{\frac{4}{a}}} p_{F}$.

where $C_{L}=3,40665 \times 10^{-\pi}$. The total energy density in terms of the nuclear, electronic, and lattice contributions is:

$\varepsilon_{\text {tot }}\left(A, Z ; \eta_{b}\right)=\frac{E(N, Z)}{A}+\frac{m_{B}^{4}}{8 \pi^{2} n_{b}}\left[x_{B} y_{B}\left(x_{B}^{2}+x_{F}^{2}\right)-\ln \left(x_{B}+y_{p}\right)\right]-C_{L} \frac{Z^{2}}{A^{4 / a}} p_{B}$.

\section{Pressure in the Outer Crust of Neutron Star}

The individual nuclei do not contribute to the pressure and, then, only the electronic and lattice terms contribute (Roca-Maza and Piekarewicz, 2008):

$P=P_{Q}+P_{L}$

$P=-\left(\frac{\partial E_{e}}{\partial V}\right)-\left(\frac{\partial E_{l}}{\partial V}\right)$

The total pressure can be written as:

$$
P\left(A, Z ; n_{b}\right)=\frac{m_{E}^{4}}{3 \pi^{2}}\left(x_{F}^{3} y_{F}-\frac{3}{8}\left[x_{F} y_{F}\left(x_{F}^{2}+y_{F}^{2}\right)-\ln \left(x_{F}+y_{F}\right)\right]\right)-\frac{n_{b}}{3} c_{L} \frac{Z^{2}}{A^{4}} p_{F} .
$$

\section{Chemical Potential in the Outer Crust of Neutron Star}

Equilibrium of system can be determined by maintaining temperature, pressure and chemical potential continuous in the outer crust. At zero temperature, chemical potential can be obtained by calculating Gibbs free energy per nucleon by a Legendre transform $(G=E+P V)$ :

$\mu(A, Z ; P)=\frac{G(A, Z ; P)}{A_{\text {tot }}}=\varepsilon\left(A_{s} Z ; n_{b}\right)+\frac{P}{n_{b}}$

$=\frac{E\left(A_{v} Z\right)}{A}+\frac{Z}{A} \mu_{\theta}-\frac{4}{3} C_{L} \frac{Z^{2}}{A^{\frac{4}{a}}} p_{F}$

where $\mu_{\varepsilon}=\sqrt{p_{F \theta}^{2} c^{2}+m_{\varepsilon}^{2} c^{4}}=\sqrt{p_{F \theta}^{2}+m_{\varepsilon}^{2}}$ is chemical potential of electrons.

We can see that the chemical potential is a function of the pressure whereas the energy per baryon is a function of the baryon density. Actually, it is convenient to compute the composition of the outer crust by minimizing the chemical potential at constant pressure rather than by minimizing the energy per particle at constant baryon density. This is because the pressure and chemical potential in the outer crust tend 
to continuous and change monotonously, whereas it does not imply the same for the baryon density and energy. The equilibrium conditions demand the pressure and chemical potential to be continuous throughout the outer crust but this does not necessarily imply the same for the baryon density and energy. Because of that, the approximation to transform energy density as function of the pressure, as well as chemical potential, was required. This approximation will be explained at the next subchapter.

Liquid Drop Model to Investigating the Outer Crust Model be applied with two assumptions. First, liquid drop model (LDM) will be used to calculate energy of nuclear. The liquid-drop mass formula (by assumsing $Z(Z-1) \approx Z^{2}$ ) may be written as (Roca-Maza and Piekarewicz, 2008)

$\varepsilon(x, y)=m_{p} y+m_{n}(1-y)-a_{v}+\frac{a_{s}}{x}+a_{c} x^{2} y^{2}+a_{a}(1-2 y)^{2}$,

Where $x=A^{1 / 3}$ and $y=Z / A$ is proton fraction (or electron). The various empirical constants $\left(a_{v}, a_{s}, a_{c}\right.$, and $a_{a}$ ) represent volume, surface, Coulomb, and asymmetry contribution, respectively (Roca-Maza and Piekarewicz, 2008):

$\begin{array}{ll}a_{v}=15,71511 \mathrm{MeV}, & a_{c}=0,71363 \mathrm{MeV}, \\ a_{s}=17,53638 \mathrm{MeV}, & a_{a}=23,37837 \mathrm{MeV} .\end{array}$

Optimal value of $x$ and $y$ can be calculated by setting both derivatives equal to zero,

$$
\begin{aligned}
& \left(\frac{\partial \varepsilon_{n}}{\partial x}\right)_{y}=-\frac{a_{g}}{x^{2}}+2 a_{c} x y^{2}=0, \\
& \left(\frac{\partial \varepsilon_{n}}{\partial y}\right)_{x}=-\Delta m+2 a_{c} x^{2} y-4 a_{a}(1-2 y)=0,
\end{aligned}
$$

where $\Delta m=m_{n}-m_{p}$. The more stable nucleus:

$A=x^{a}=\left(\frac{a_{g}}{2 a_{e}}\right) \frac{1}{y^{2}}$

$y=\frac{1+\left(\frac{\Delta m}{4 a_{a}}\right)}{2+\left(\frac{a_{c}}{2 a_{a}}\right) x^{2}} \approx \frac{1 / 2}{1+\left(\frac{a_{c}}{4 a_{a}}\right) x^{2}}$.

If both equation are solved, then one finds the most stable nucleus with $x_{0}=3,90611$ and $y_{0}=0,45405$, or, equivalently, $A_{0}=59,59869 ; Z_{0}=27,06062 ; N_{0}=$ 32,53807 .

Second assumption is the electronic contribution will be assumed to be that of an extremely relativistic (i.e., $m_{e} / p_{\mathrm{Fe}} \rightarrow 0$ ) Fermi gas, so the energy density of electron may be written as:

$\varepsilon_{\theta} \approx \frac{3}{4} y^{\frac{4}{3}} p_{F}$

With this assumption, equations of total energy density, chemical potential and pressure can be written as,

$$
\begin{aligned}
& \varepsilon\left(x, y, p_{F}\right)=\varepsilon_{n}(x, y)+\frac{3}{4} y^{\frac{4}{3}} p_{F}-C_{L} x^{2} y^{2} p_{F} \\
& \mu\left(x, y, p_{F}\right)=\varepsilon_{n}(x, y)+y^{\frac{4}{3}} p_{F}-\frac{4}{a} C_{L} x^{2} y^{2} p_{F} \\
& P\left(x, y, p_{F}\right)=\frac{n_{b}}{4} y^{\frac{4}{3}} p_{F}-\frac{n_{b}}{3} C_{L} x^{2} y^{2} p_{F} .
\end{aligned}
$$

At the predicted neutron drip density of about $4 \times 10^{11}$ $\mathrm{g} \mathrm{cm}^{-3}$, the Fermi momentum is approximately equal to $p_{F}{ }^{\max } \approx 40 \mathrm{MeV}$ and contribution of electrons at equation (3.18) will have the energy about $\varepsilon_{e}{ }^{\max } \approx 30 y^{4 / 3}$ (Roca-Maza and Piekarewicz, 2008). Incorporating electronic and lattice contributions to the semiempirical mass formula yield the following expression for the total energy per nucleon of the system (RocaMaza and Piekarewicz, 2008):

$$
\begin{aligned}
& \varepsilon\left(x_{s} y_{s} p_{p}\right)=m_{p} y+m_{n}(1-y)-a_{v}+\frac{a_{x}}{x}+ \\
& a_{c} x^{2} y^{2}+a_{a}(1-2 y)^{2}+\frac{a}{4} y^{\frac{4}{3}} p_{p}-C_{L} x^{2} y^{2} p_{p}
\end{aligned}
$$

As done before for the pure nuclear contribution, the optimal values of $x$ and $y$-at fixed density-may be obtained by setting both derivatives equal to zero. That is,

$\left(\frac{\partial \varepsilon}{\partial x}\right)=-\frac{a_{g}}{x^{2}}+2 \widetilde{a}_{c} x y^{2}=0$,
$\left(\frac{\partial \varepsilon}{\partial y}\right)_{x_{i} p_{F}}=-\Delta m+2 \widetilde{a}_{c} x^{2} y-4 a_{a}(1-2 y)+y^{\frac{1}{1} p_{F}}=0$.

where $\tilde{a}_{c}\left(p_{F}\right) \equiv\left(a_{c}-C_{L} p_{F}\right)$.

\section{First-Order Solution}

To demonstrate how free electron and lattice impact to stable nuclei, an approximation first-order solutions for equations (3.22a) and (3.22b) have been calculated (Roca-Maza and Piekarewicz, 2008):

$x\left(p_{F}\right)=x_{0}(1+\xi)$ and $y\left(p_{F}\right)=y_{0}(1+\eta)$

where both $\xi$ and $\eta$ represent small (i.e., first-order in $\left.p_{F}\right)$ deviations from the zero-density results. Substituting these equations into Equation. (3.22a) and (3.22b) yields the first-order solutions. One obtains:

$$
\begin{aligned}
& x\left(p_{F}\right)=x_{0}\left[1+\left(\frac{\left(C_{1}-1\right) C_{L}+2 C_{2}}{3 C_{1}-1}\right) \frac{p_{F}}{a_{c}}\right] \\
& =\left(3,90610+0,03023 p_{F}\right), \\
& y\left(p_{F}\right)=y_{0}\left[1-\left(\frac{3 C_{2}-C_{L}}{3 C_{1}-1}\right) \frac{p_{F}}{a_{e}}\right] \\
& =\left(0,45405-0,00419 p_{F}\right),
\end{aligned}
$$

where $C_{1}$ and $C_{2}$ are dimensionless quantities

$$
\begin{aligned}
& C_{1}=\frac{4 a_{a}}{x_{0}^{2} a_{c}} \approx 8,58843 \\
& C_{2}=\frac{1}{2 x_{0}^{2} y_{0}^{\frac{2}{3}}} \approx 0,05547 .
\end{aligned}
$$




\section{Exact Solutions}

Exact solutions are calculated to find roots of equations (3.22a) and (3.22b) (Roca-Maza and Piekarewicz, 2008):

$$
\begin{aligned}
& x(y)=\left(\frac{a_{g}}{2 y^{2} \tilde{a}_{c}}\right)^{\frac{1}{2}}, \\
& p_{F}(y)=\frac{\Delta m-2 \tilde{a}_{c} x^{2} y+4 a_{a}(1-2 y)}{y^{\frac{1}{a}}-2 C_{L} x^{2} y} .
\end{aligned}
$$

By using substitution method, the composition of outer crust is nuclei with $\mathrm{Z} \approx 20-50$ region. From these solutions, nucleon number $x=A^{1 / a}$ and fraction proton $y=Z / A$ as function of momentum can be plotted as:
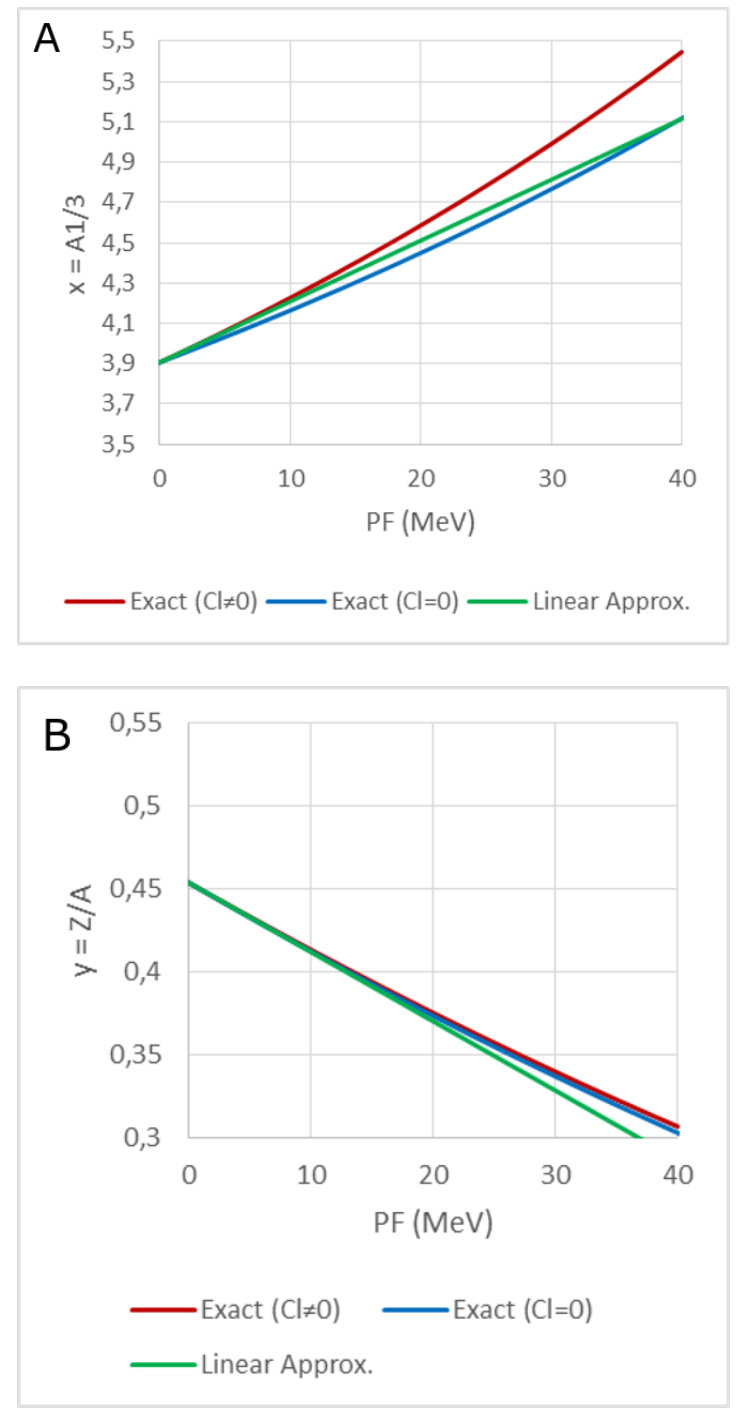

Figure 1. Baryon number $\mathrm{x}=\mathrm{A}^{1 / 3}(\mathrm{~A})$ and Proton fraction $\mathrm{y}=\mathrm{Z} / \mathrm{A}(\mathrm{B})$ displayed as a function of the Fermi momentum $p_{F} \equiv\left(3 \pi^{2} \mathrm{n}_{\mathrm{b}}\right)^{1 / 3}($ Roca Maza and Piekarewicz, 2008).

In figure 1, the nucleon number and the proton fraction are displayed as a Fermi momentum function. Green curve lines represent first-order linear solutions while blue and red curves represent exact solutions when $C_{L}=0$ and $C_{L} \neq 0$. When exact solutions $C_{L} \neq 0$ are combined (lattice contribution is involved), the deviation of the value of the solution becomes greater. This shows that even though the lattice contribution is small, it shifts the equilibrium core to a larger nucleon number.

In the uppermost layer of the outer crust, the proton fraction $y$ is equal to 0,$45200 ; 0,45405$ and 0,45405 for exact solutions when $C_{L}=0$, and $C_{L} \neq 0$ and linear solutions. This value has a slight difference from the proton Fe fraction $(0,46429)$. With this solution, the neutron drip line is $y_{\text {drip }}=0,30400 ; 0,30655$ and 0,28645 for exact solutions when $C_{L}=0$, and $C_{L} \neq 0$ and linear solutions. These values are slightly different from the proton fraction $\mathrm{Kr}(0,30508)$ which is a conventionally accepted core drop. With these results, it can be estimated that the core in the outer layer of the outer crust corresponds to the core ${ }^{154} \mathrm{Cd}$. However, from these three solutions, it has been successfully demonstrated that a mass model in the form of a liquid drop model can be used to determine the composition of the cores in the outer crust (Roca-Maza and Piekarewicz, 2008).

In addition to the proton fraction and nucleon number, the pressure in the outer crust layer can also be displayed as a momentum function:

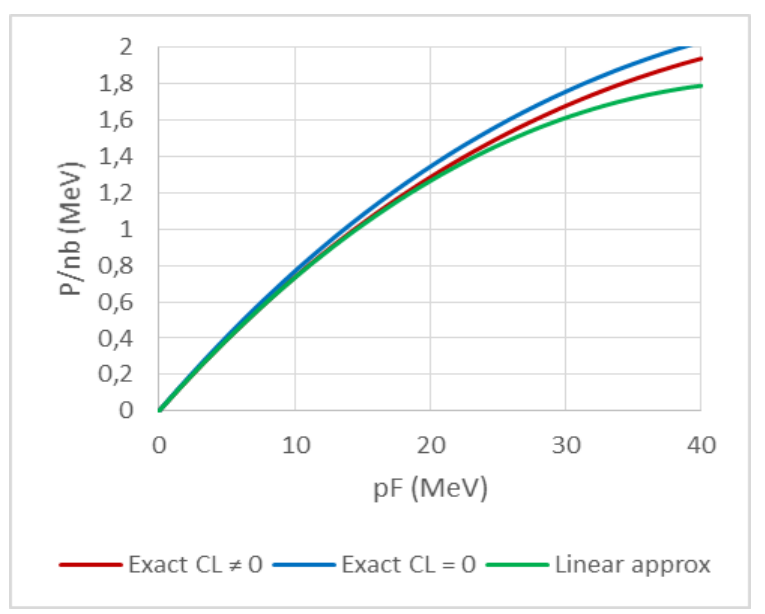

Figure 2. Pressure as a function of the Fermi momentum $p_{F} \equiv\left(3 \pi^{2} n_{b}\right)^{1 / 3}$ (Roca-Maza and Piekarewicz, 2008).

From figure 2, it is known that the pressure in the outer crust increases smoothly as a function of depth. In other words, pressure increases with increasing density in the outer crust layer. The equation for first-order pressure can be written as (Roca-Maza and Piekarewicz, 2008):

$$
\frac{P}{n_{b}}\left(x, y, p_{F}\right)=\frac{1}{4} y^{\frac{4}{3}} p_{F}-\frac{1}{3} C_{L} x^{2} y^{2} p_{F}
$$

\section{Discussion}

Following the work by Baym, Pethick, and Sutherland, as well as the more recent work by Roca-Maza and Piekarewicz, we studied the composition and equation of state of the outer crust of non-accreting neutron 
stars. The outer crust consists of ions $Z$ which arranged in crystal Coulomb and free electrons $e$. The principal contributions to the energy of matter in this regime are the energy density of nuclear, the energy density of free electron, and the energy density of lattice. Liquid drop model is used to calculate nuclear energy and free electron is assumed relativistic. With a simple model based on liquid drop model, it is known that the outer crust is composed of nuclei which are at $\mathrm{Z} \approx 20-50$, with ${ }^{56} \mathrm{Fe}$ core at the upper layer, and ${ }^{154} \mathrm{Cd}$ core at the lowermost layer of the outer crust. The equation of state (the relationship between pressure and density) shows that the pressure increases with increasing density in the outer crust layer.

\section{CONCLUSIONS}

Based on the research it can be concluded that, in figure 1 , the nucleon number and the proton fraction are displayed as a Fermi momentum function. Green curve lines represent first-order linear solutions while blue and red curves represent exact solutions when $C_{L}=0$ and $C_{L} \neq 0$. When exact solutions $C_{L} \neq 0$ are combined (lattice contribution is involved), the deviation of the value of the solution becomes greater. This shows that even though the lattice contribution is small, it shifts the equilibrium core to a larger nucleon number.

From figure 2, it is known that the pressure in the outer crust increases smoothly as a function of depth. In other words, pressure increases with increasing density in the outer crust layer.

The research was study the composition and equation of state of the outer crust of non-accreting neutron stars. The outer crust consists of ions $Z$ which arranged in crystal Coulomb and free electrons $e$. The principal contributions to the energy of matter in this regime are the energy density of nuclear, the energy density of free electron, and the energy density of lattice.

With a simple model based on liquid drop model, it is known that the outer crust is composed of nuclei which are at $\mathrm{Z} \approx 20-50$, with ${ }^{56} \mathrm{Fe}$ core at the upper layer, and ${ }^{154} \mathrm{Cd}$ core at the lowermost layer of the outer crust. The equation of state (the relationship between pressure and density) shows that the pressure increases with increasing density in the outer crust layer.

\section{ACKNOWLEDGMENTS}

F.F., E.T.S, R.H.S.B are grateful to I. Santosa and J. Partini for comments, to all research member of Astrophysics, Cosmology and Mathematical Physics Group, and to all laboratory assistant of Physics, Faculty of Mathematics and Natural Science.

\section{REFERENCES}

Arya, Atam. P. 1966. Fundamental of Nuclear Physics. West Virginia University. Boston.

Baade, W., and Zwicky, F. 1934. Remarks on Supernovae and Cosmic Rays, Phys. Rev. 46: 76.

Baym, G., Pethick, C. and Sutherland, P. 1971. The Ground State of Matter at High Densities: Equation of State and Stellar Models. Astrophys. J 170: 299 - 317.

Chamel, N. 2007. Neutron Star Crust Beyond The Wigner-Seitz Approximation. arXiv:0709.3798v1: 1-8.

Chamel, N. and Haensel, P. 2008. Physics of Neutron Star Crusts. Living Rev. Relativity, 11: 10.

Haensel, P. 1995. Solid Interiors of Neutron Stars and Gravitational Radiation, arxiv.org/abs/astro-ph/9605164: 1-26.

Haensel, P., Potekhin, A.Y. and Yakovlev, D.G. 2007. Neutron Stars 1 Equation of State and Structure. Springer. New York.

Istiqomah, E. L. 2010. Critical Temperatures and Energy Gaps Superfluid on The Cooling Neutron Stars Core. [Thesis]. Gadjah Mada University. Yogyakarta. [Indonesian

Kusminarto. 2011. Esensi Fisika Modern. Andi Publisher. Yogyakarta. [Indonesian]

Lander, S. K. 2010. Equlibria and Oscillations of Magnetised Neutron Stars. [Thesis]. University Of Southamto

Meyerhof, Walter E. 1967. Elements of Nuclear Physics. Stanford University. McGRAW-HILL Book Company. New York

Roca-Maza, X. and Piekarewicz, J. 2008. Impact of the symmetry energy on the outer crust of non-accreting neutron stars. Phys. Rev. C78: 025807.

Roca-Maza. 2010. Isospin asymmetry in stable and exotic nuclei. [Thesis]. Department Estructura i Consitituents de la Materia. Universitat de Barcelona.

Shapiro, S. L. and Teukolsky, T. A. 1983. Black Holes, White Dwarf, Neutron Stars. John Wiley \& Sons Inc. New York. 
THIIS PAGE INTENTIONALLY LEFT BLANK 